\title{
POSEIDON 1 and 2: Probable Causes and Proposed Treatment Strategies? An Evidence-based Update
}

\author{
Atri Pal ${ }^{1}$, Mohan S Kamath ${ }^{2}$
}

\begin{abstract}
Aim and objective: To elucidate the cause of poor ovarian response to controlled ovarian hyperstimulation during in vitro fertilization in women with good ovarian reserve and the potential treatment options for them.

Background: There has been a steady increase in number of in vitro fertilization (IVF) cycles being performed across the world. An important step of IVF is controlled ovarian hyperstimulation $(\mathrm{COH})$, with an aim to achieve multifollicular response. Conventionally the protocol and gonadotropin dose is tailored to ensure adequate oocyte yield and minimize complications. Studies suggest that maximizing oocyte yield increases the cumulative LBR. However, in spite of high dose of gonadotropin usage during $\mathrm{COH}$, many women have poor response (<4 oocytes retrieved) and/or low oocyte yield (4-9 oocytes retrieved). Patient-Oriented Strategies Encompassing Individualize D Oocyte Number (POSEIDON) criteria to classify poor responders were introduced in 2016 to achieve better stratification of poor responders and achieve an individualized treatment approach for the patients.

Review results: Some of the proposed reasons include suboptimal gonadotropin dose, gonadotropin receptor resistance due to gonadotropin receptor polymorphism and issues with ovulation trigger. Two most studied single nucleotide polymorphism are those at position 307 and 680 of exon 10 of Follicle stimulating hormone receptor. Some studies have demonstrated that homozygous Asparagine at position 680 required lesser gonadotropin dose and had more oocyte yield in normoovulatory women compared with other variants at position 680. However, other studies have reported contradictory findings. Similarly contradictory results have been reported from various studies regarding ovarian response with respect to variants at locus 307 . Some of the proposed treatments for patients with unexpected responders include increasing the dose of Inj. FSH, adding Inj. Luteinizing hormone receptor (LH) to ovarian stimulation, use of dual trigger, synchronizing the follicular cohort, use of adjuvants during IVF, and dual stimulation.

Conclusion: The exact reason for such a response is still unclear although role of FSH/LH polymorphism has been studied extensively. However, no specific FSH/LH polymorphism has been consistently been associated with such unexpected hyporesponse. There is no high quality evidence for other proposed treatment options.

Keywords: BOLOGNA criteria, Dual trigger, FSH receptor polymorphism, LH receptor polymorphism, Poor ovarian responders, POSEIDON classification.

International Journal of Infertility and Fetal Medicine (2022): 10.5005/jp-journals-10016-1257
\end{abstract}

\section{BACKGROUND}

It has been more than 40 years since the birth of the first in vitro fertilization (IVF) baby in Cambridge, UK ${ }^{1}$ and subsequently, more than 8 million babies have been born following IVF. ${ }^{1}$ In many parts of the world, there is a trend which suggests that more couples are delaying the decision to start a family. ${ }^{2}$ As a result, there has been an increase in the mean age at which the woman delivers her first baby. ${ }^{3}$ There has been a steady increase in number of IVF cycles being performed across the world. ${ }^{4,5}$ For many of the infertile couples due to various reasons such as advanced age, diminished ovarian reserve, severe tubal factors, and male factor infertility, assisted reproduction technology (ART) remains the most effective treatment option.

An important step of IVF is controlled ovarian hyperstimulation $(\mathrm{COH})$, with an aim to achieve multifollicular response. ${ }^{6}$ Conventionally, the protocol and gonadotropin dose is tailored according to the woman's age, body mass index, presence of polycystic ovaries morphology on ultrasound, ovarian reserve and previous ovarian response to $\mathrm{COH}$ to ensure adequate oocyte yield, and minimize complications such as OHSS. ${ }^{7}$ Earlier studies have reported that the live birth rate per fresh embryo transfer continues to rise with increase in number of oocytes retrieved but it plateaus when approximately 15 oocytes are obtained following oocyte retrieval. However, recent studies have reported that cumulative

\footnotetext{
1,2Department of Reproductive Medicine, Christian Medical College, Vellore, Tamil Nadu, India

Corresponding Author:Mohan S Kamath, Department of Reproductive Medicine, Christian Medical College, Vellore, Tamil Nadu, India, Phone: +919994497690, e-mail: dockamz@gmail.com
}

How to cite this article: Pal A, Kamath MS. POSEIDONE 1 and 2: Probable Causes and Proposed Treatment Strategies? An Evidencebased Update. Int J Infertil Fetal Med 2022;13(1):23-27.

Source of support: Nil

Conflict of interest: None

LBR continues to increase with oocyte yield beyond 15 oocytes. ${ }^{8,9}$ These studies suggest that maximizing oocyte yield increases the cumulative LBR and indirectly suggest aggressive ovarian stimulation to achieve the goal.

However, in spite of high dose of gonadotropin usage during $\mathrm{COH}$, many women have poor ovarian response $(<4$ oocytes retrieved) and are called poor responders. Poor ovarian response is usually seen in women with advanced age, diminished ovarian reserve and in some cases, young women with adequate ovarian reserve. The prevalence of poor responders varies between 5.6 and $35.1 \% .{ }^{10,11}$ Though a number of interventions have been suggested, 
most have been found to be of little benefit in improving treatment outcomes. ${ }^{12,13}$

\section{Bologna Criteria}

Initially, the definition of poor responders lacked standardization which led to surfacing of heterogeneity issues when evaluation of intervention for this population was contemplated. ${ }^{14}$ In the last decade, there have been efforts to standardize the definition. To address the problem, and as a first step, Bologna criteria were introduced in 2011 after expert consensus. ${ }^{15}$ According to Bologna criteria, poor responders are identified based on age ( $\geq 40$ years), history of poor response in previous cycle ( $\leq 3$ oocytes) and/or abnormal ovarian reserve test.

However, Bologna criteria had its own shortcomings. In the Bologna criteria, the cut off age is $\geq 40$ years. It has been seen that women with age $<40$ years is a very broad category and they can be further subdivided into different age-groups based on different prognosis to reduce heterogeneity. ${ }^{16}$ Another important drawback is the wide range of the cut off values of the ovarian reserve test. $^{17}$ The accuracy and validity of this wide range of value was not clearly explained by ESHRE consensus. ${ }^{17}$ The subpopulation of young women with adequate ovarian reserve but unexpected poor response or suboptimal ovarian response (4-9 oocytes retrieved) were not addressed in Bologna criteria. Embryo ploidy, which depends on quality of the oocyte, which in turn is influenced by age of the woman (aneuploidy increasing with women age $>35$ years), goes unanswered under the Bologna criteria. ${ }^{18}$

\section{POSEIDON Classification}

To deal with the limitations of Bologna criteria, The Patient-Oriented Strategies Encompassing Individualize D Oocyte Number (POSEIDON) group was established in 2016 comprising of experts from the field of Reproductive Medicine, from various countries. ${ }^{19}$ They first introduced the concept of low response and suboptimal ovarian response. According to this system the patients were classified into four groups. ${ }^{19}$

Young women, less than 35 years, with adequate ovarian reserve but with a history of poor ( $<4$ oocytes retrieved) or suboptimal ovarian response (4-9 oocytes retrieved) in a previous IVF cycle were classified as Group 1 while women aged 35 years or more with adequate ovarian reserve and similar history have been classified separately in Group $2 .{ }^{19}$ Group 3 and 4 include women with diminished ovarian reserve aged less than 35 years and 35 or more years, respectively. POSEIDON concept attempted to achieve a more pragmatic goal: (1) a better stratification of the so called low prognosis women, (2) an individualized treatment approach for the patients. ${ }^{19}$

Out of the four groups in POSEIDON classification, Group 1 women and to an extent, Group 2 as well, who had started an IVF cycle with good prognosis but had an unexpected poor or suboptimal response have been focus of ongoing research. The unexpected poor/suboptimal response leads to cycle cancellations, and/or lower LBRs which increases the treatment burden for the couples and is frustrating for the clinicians. There is a need for greater understanding of the probable reason for such a response which can help plan treatment strategies and optimize the outcomes in these groups.

\section{Causes for POSEIDON 1 and 2}

The probable cause and pathophysiological mechanism of unexpected poor response to ovarian stimulation is not yet clear. ${ }^{20}$ Some of the proposed reasons include suboptimal gonadotropin dose, gonadotropin receptor resistance due to gonadotropin receptor polymorphism and issues with ovulation trigger. $^{21}$

Follicle stimulating hormone (FSH) receptor/Luteinizing hormone receptor (LH) polymorphism:

Mutation and polymorphism are the existence of more than one variant, with respect to chromosomal structure, gene and protein sequence. ${ }^{22}$ When the variations occur with frequency of $\geq 1 \%$ in the general population, it is known as polymorphism. ${ }^{23}$ Polymorphism can involve changes in one or more nucleotide. Single nucleotide polymorphism (SNP) is the most common. It can be used as genetic signature to study various traits and diseases in the population.

Studies have reported single nucleotide polymorphisms at various loci in the coding region of FSH receptor. The two most studied SNPs are those at position 307 and 680 of exon $10 .{ }^{24}$ Polymorphism at position 307 results in presence of either threonine or alanine and at position 680 it results in either asparagine or serine. At locus 307 the possible variants are homozygous alanine (Ala ${ }^{307} \mathrm{Ala}$ ), homozygous threonine ( $\mathrm{Thr}^{307} \mathrm{Thr}$ ) or heterozygous alanine-threonine (Ala ${ }^{307} \mathrm{Thr}$ ). At position 680 the possible variants are homozygous serine (Ser ${ }^{680}$ Ser), homozygous asparagine $\left(A s p{ }^{680} \mathrm{Asp}\right)$ or heterozygous serine-asparagine (Ser ${ }^{680} \mathrm{Asp}$ ). In the LH receptor, the most studied polymorphism is the one at locus 312 (between Asparagine and serine). ${ }^{25}$

Studies have reported that the response of ovarian stimulation with gonadotropins is related to $\mathrm{FSH}$ receptor gene polymorphism but results from various studies are conflicting. ${ }^{26,27}$ Mayorga et al. and Yan et al. demonstrated that patients with homozygous Asparagine at position 680 required lesser gonadotropin dose and had more oocyte yield in normoovulatory women. ${ }^{26,28}$ Contradicting this, Behre et al. and Achrekar et al. demonstrated in normogonadotropic ovulatory women when similar dose of FSH is administered, there was no statistically significant difference in number of oocytes retrieved, clinical pregnancy rate in different variants at locus $680 . .^{24,29}$ Similarly Laven et al. showed from their study in WHO class II anovulatory infertile women that FSHR polymorphism at 680 did not have significant effect on the amount of FSH used. ${ }^{30}$

With respect to polymorphism at 307 , an earlier study reported that women with homozygous Alanine at position 307 required lower amount of FSH and those with homozygous for Threonine required higher amount of FSH dose during ovarian stimulation. ${ }^{24}$ On the contrary Yan et al. reported that in couples with either tubal or male factor infertility, women with Ala307Ala had significantly higher proportion of poor response. ${ }^{31}$ Laven et al. found that FSHR polymorphism at 307 did not have significant effect on the amount of FSH used ${ }^{30}$ in women with WHO class II ovulatory dysfunction.

With respect to FSH receptor polymorphism and basal serum FSH level, Yan et al. reported that at locus 307 homozygous Alanine had a significantly higher basal FSH compared to the other two variants ${ }^{31}$ However, the Indian study by Achrekar et al. found no significant difference in basal FSH among the different variants at 307 or $680 . .^{24}$ Similar findings with respect to position 307 was reported by Dolfin et al. ${ }^{32}$ With respect to FSH receptor polymorphism at locus 680 , basal FSH was significantly more in Ser ${ }^{680}$ Ser compared to the other two variants in various other studies. ${ }^{26,28,30,31}$

There is paucity of studies evaluating the role of $\mathrm{LH}$ receptor polymorphism. Most studies involved $\mathrm{LH}$ receptor at polymorphism at 312. Lindgren et al. reported no difference in 
oocyte numbers when different variants were compared but the pregnancy rate was significantly higher in homozygous Serine at position 312 compared to the heterozygous serine, threonine, or homozygous threonine. ${ }^{25}$

\section{Proposed Treatment Strategies for POSEIDON 1 and 2}

Unlike women of other two POSEIDON groups, women in POSEIDON Group 1 and Group 2 have the advantage of having adequate ovarian reserve. Therefore, a properly tailored IVF cycle will help in achieving adequate number of oocytes and thereby improve treatment outcome.

Increasing the dose of FSH: Since one of the proposed mechanisms for unexplained hypo-response is FSH receptor polymorphism, rendering FSH receptor resistant to the gonadotropin, one of the option is to increase the starting dose of FSH used for ovarian stimulation for subsequent ART cycle. A recent systematic review reported that the chance of poor response was lower and oocyte yield was significantly higher with gonadotropin dose of $300 \mathrm{IU}$ compared to $150 \mathrm{IU} .{ }^{33}$ An increase in starting dose of gonadotropin dose in women with previous history of poor/suboptimal response may result in optimum treatment outcome. ${ }^{34}$

Addition of LH: Considering that $\mathrm{LH}$ receptor polymorphism may be a cause of hyporesponse, adding $\mathrm{LH}$ along with FSH during ovarian stimulation can be beneficial. A recent meta-analysis reported that adding $\mathrm{LH}$ to FSH during ovarian stimulation, the number of oocytes retrieved, implantation rate, clinical pregnancy rate, and live birth rate increased significantly. ${ }^{35}$ Similar conclusions were made by Alviggi et al. in their systematic review. ${ }^{36}$ However in most of the studies included in the review, the protocol used was long protocol and the definition of poor responders was neither homogenous nor standardized. Studies specific to POSEIDON 1 or 2 patients are lacking.

Dual trigger: Dual trigger is considered to be more physiological, by providing both the FSH and LH surge unlike the conventional single ovulation triggers. Earlier studies have shown that dual trigger help in increasing the number of mature oocytes in case of normorespnders and hyperresponders. ${ }^{37,38}$ Recent retrospective cohort studies ${ }^{39,40}$ reported an increase in the total number of oocytes retrieved, number of mature oocytes retrieved and higher number of cleavage stage embryos in women with diminished ovarian reserve following administration of dual trigger compared to trigger with only Inj. hCG. However, two recent RCTs did not demonstrate any benefit of dual trigger over Inj. hCG alone trigger in poor responders. ${ }^{41,42}$ Khalife et al. conducted an interim analysis of their study comparing dual trigger using triptorelin and hCG vs only hCG in Bologna criteria poor responders. They concluded that the total number of oocytes retrieved, number of mature oocytes, and fertilization rate was not significantly different between the two groups. ${ }^{42}$ However studies evaluating women belonging to POSEIDON Group 1 or 2 are lacking.

Synchronizing the follicle cohort: In antagonist protocols risk of asynchronous development of follicle cohort is high, leading to a low Follicle output ratio (FORT) and this may lead to suboptimal/poor oocyte yield. Oral contraceptive pills and luteal phase estrogen have been widely used to synchronize the follicle cohort and cycle programming. OC pill can be used to synchronize the follicular cohort in antagonist cycles. However, recent Cochrane meta-analysis has shown that number of oocytes retrieved was not statistically different in OCP pretreated antagonist cycle compared to antagonist cycle without pretreatment with $\mathrm{OCP}$, and the live birth rate was significantly lesser in OCP pretreated group..$^{43}$ Option of pretreatment with estrogen can be explored in antagonist cycle for follicular synchronization without any impact on Live birth rate.

Adjuvants during IVF stimulation: Adjuvants like androgens (DHEA and testosterone) and growth hormones are offered to improve the outcome of IVF treatment. However, most of these interventions lack robust evidence and are expensive. A recent metanalysis ${ }^{44}$ evaluating the role of Growth hormone in poor responders reported that there was an improvement in total number of oocytes, number of mature oocytes retrieved, number of embryos available, and clinical pregnancy rate. However, there was no significant improvement in live birth rate. Subgroup analysis of studies using Bologna criteria to define poor responders could not confirm improvement in clinical pregnancy rate. Moreover an appropriate dose, regimen, and protocol for growth hormone remains unclear. Studies have used reported use of Growth hormone 1 IU every alternate day to 12 IU daily. A Cochrane systematic review concluded that pretreatment with testosterone and DHEA in poor responders undergoing IVF, may be associated with improvement in live birth rate. ${ }^{45}$ However, there is insufficient evidence to conclude regarding safety of these drugs and controversies regarding their beneficial effect still persist. Further studies are required for definitive conclusion regarding their benefit and safety and and also to the dose and regimen in POSEIDON Group 1. ${ }^{46}$

Dual stimulation: Dual stimulation, in which ovarian stimulation is conducted in follicular phase and luteal phase of the same cycle has been proposed as an option to increase the oocyte yield in a single ovarian cycle. ${ }^{47}$ Studies have demonstrated that the oocytes retrieved in luteal phase is comparable to the oocytes retrieved in follicular phase with respect to fertilization, euploidy, and blastulation rate. ${ }^{48} \mathrm{~A}$ case series of 54 bologna criteria poor responders reported that the number of oocytes retrieved in luteal phase stimulation was more compared to that in follicular phase stimulation. ${ }^{49}$ Dual stimulation may help in decreasing the time to obtain a single euploid embryo for transfer and decrease drop out rate. ${ }^{48}$ However, this stimulation protocol requires mandatory elective cryopreservation. Further studies regarding the safety of dual stimulation are required before it can be advocated routinely. Studies regarding the effectiveness of dual stimulation in POSEIDON Group 1 and 2 patients are lacking.

\section{Discussion}

In recent times significant effort has been made to standardize the concept of poor responders and women with low prognosis. The introduction of POSEIDON criteria has helped in understanding and stratifying women with expected poor response and those with unexpected poor response. One of the most important groups of women is those who have adequate ovarian reserve but end up with unexpected poor-response/suboptimal response. The exact reason for such a response is still unclear although role of $\mathrm{FSH} / \mathrm{LH}$ polymorphism has been studied extensively. However, no specific FSH/LH polymorphism has been consistently associated with such unexpected hyporesponse. Thus, role of FSH/LH receptor polymorphism testing in women with poor/suboptimal response is unclear. In majority of the ART cycles with history of poor/suboptimal response, the clinicians increase gonadotropin starting dose or change the protocol which most often result in increased oocyte yield and thereby an increase in LBR. There is 
no high quality evidence for other proposed treatment options such as addition of recombinant LH and duo stimulation which also increase the cost and are not patient friendly. OC pill/estradiol pretreatment can be explored in antagonist protocol for better follicular synchronization but there is ongoing concern about negative impact of OC pill on LBR in antagonist cycle. Dual trigger seems to be upcoming strategy and early studies indicate higher oocyte yield.

There is need to plan a study to determine the prevalence of FSH/LH polymorphism in different subpopulation of POSEIDON to establish its role in gonadotropin response during ovarian stimulation.

\section{Conclusion}

Unexpected poor responders are an important group of patients in our clinical practice. The exact cause of the poor ovarian response is not properly understood but gonadotropin receptor polymorphism can be a plausible explanation. Various treatment options have been proposed for this group of patients, however robust evidence is lacking. Further research is required to elucidate the cause of such poor/suboptimal response and the role of FSH receptor polymorphism in such cases. Further well designed RCTs are required to determine the treatment strategies of this group of women under POSEIDON 1 and 2.

\section{References}

1. Yovich JL. Founding pioneers of IVF update: innovative researchers generating livebirths by 1982. Reprod Biol 2020;20(1):111-113. DOI: 10.1016/j.repbio.2019.12.008

2. Mills M, Rindfuss RR, McDonald P, et al. Why do people postpone parenthood? Reasons and social policy incentives. Hum Reprod Update 2011;17(6):848-860. DOI: 10.1093/humupd/dmr026

3. de Graaff AA, Land JA, Kessels AG, et al. Demographic age shift toward later conception results in an increased age in the subfertile population and an increased demand for medical care. Fertil Steril 2011;95(1):61-63. DOI: 10.1016/j.fertnstert.2010.05.013

4. De Geyter C, Calhaz-Jorge C, Kupka MS, et al. ART in Europe, 2015: results generated. Hum Reprod Open 2020;2020(1):hoz038. DOI: 10.1093/hropen/hoz038

5. Malhotra N, Shah D, Pai R, et al. Assisted reproductive technology in India: a 3 year retrospective data analysis. J Hum Reprod Sci 2013;6(4):235-240. DOI: 10.4103/0974-1208.126286

6. Fauser BC, Devroey P, Yen SS, et al. Minimal ovarian stimulation for IVF: appraisal of potential benefits and drawbacks. Hum Reprod 1999;14(11):2681-2686. DOI: 10.1093/humrep/14.11.2681

7. La Marca A, Sunkara SK. Individualization of controlled ovarian stimulation in IVF using ovarian reserve markers: from theory to practice. Hum Reprod Update 2014;20(1):124-140. DOI: 10.1093/humupd/dmt037

8. Polyzos NP, Drakopoulos P, Parra J, et al. Cumulative live birth rates according to the number of oocytes retrieved after the first ovarian stimulation for in vitro fertilization/intracytoplasmic sperm injection: a multicenter multinational analysis including $\sim 15,000$ women. Fertil Steril 2018;110(4):661-670. DOI: 10.1016/j.fertnstert.2018.04.039

9. Zhao Z, Shi H, Li J, et al. Cumulative live birth rates according to the number of oocytes retrieved following the "freeze-all" strategy. Reprod Biol Endocrinol 2020;18(1):14. DOI: 10.1186/s12958-020-00574-3

10. Oudendijk JF, Yarde F, Eijkemans MJ, et al. The poor responder in IVF: is the prognosis always poor?: a systematic review. Hum Reprod Update 2012;18:1-11. DOI: 10.1093/humupd/dmr037

11. Patrizio P,Vaiarelli $A$, Setti L, etal. How to define, diagnoseand treat poor responders? Responses from a worldwide survey of IVF clinics. Reprod Biomed Online 2015;30:581-592. DOI: 10.1016/j.rbmo.2015.03.002
12. Gonda KJ, Domar AD, Gleicher N, et al. Insights from clinical experience in treating IVF poor responders. Reprod Biomed Online 2018;36(1):12-19. DOI: 10.1016/j.rbmo.2017.09.016

13. Blumenfeld $Z$. What is the best regimen for ovarian stimulation of poor responders in ART/IVF?. Front Endocrinol 2020;11:192. DOI: 10.3389/fendo.2020.00192

14. Polyzos NP, Devroey P. A systematic review of randomized trials for the treatment of poor ovarian responders: is there any light at the end of the tunnel? Fertil Steril 2011;96:1058-1061. DOI: 10.1016/j. fertnstert.2011.09.048

15. Ferraretti AP, La Marca A, Fauser BC, et al. ESHRE consensus on the definition of 'poor response' to ovarian stimulation for in vitro fertilization: the Bologna criteria. Hum Reprod 2011;26:1616-1624. DOI: 10.1093/humrep/der092

16. Ke $\mathrm{H}$, Chen $\mathrm{X}$, Liu YD, et al. Cumulative live birth rate after three ovarian stimulation IVF cycles for poor ovarian responders according to the bologna criteria. J Huazhong Univ Sci Technolog Med Sci 2013; 33:418-22. DOI: 10.1007/s11596-013-1134-7

17. Esteves SC, Roque M, Bedoschi GM, et al. Defining low prognosis patients undergoing assisted reproductive technology: POSEIDON criteria-the why. Front Endocrinol 2018;9:461. DOI: 10.3389/fendo.2018.00461

18. Esteves SC, Carvalho JF, Martinhago CD, et al. Estimation of age-dependent decrease in blastocyst euploidy by next generation sequencing: development of a novel prediction model. Panminerva Med 2018;61(1):3-10. DOI: 10.23736/S0031-0808.18.03507-3

19. Humaidan P, Alviggi $C$, Fischer $R$, et al. The novel POSEIDON stratification of 'Low prognosis patients in Assisted Reproductive Technology' and its proposed marker of successful outcome. F1000Res 2016;5:2911. DOI: 10.12688/f1000research.10382.1

20. Conforti A, Esteves SC, Cimadomo D, et al. Management of women with an unexpected low ovarian response to gonadotropin. Front Endocrinol 2019;10:387. DOI: 10.3389/fendo.2019.00387

21. Alviggi C, Conforti A, Esteves SC, et al. Understanding ovarian hypo-response to exogenous gonadotropin in ovarian stimulation and its new proposed marker-the Follicle-To-Oocyte (FOI) Index. Front Endocrinol 2018;9:589. DOI: 10.3389/fendo.2018.00589

22. Daly AK. Pharmacogenetics and human genetic polymorphisms. Biochem J 2010;429(3):435-449. DOI: 10.1042/BJ20100522

23. Brookes AJ. The essence of SNPs. Gene 1999;234:177-186. DOI:10.1016/s0378-1119(99)00219-x

24. Achrekar SK, Modi DN, Desai SK, et al Follicle-stimulating hormone receptor polymorphism (Thr307Ala) is associated with variable ovarian response and ovarian hyperstimulation syndrome in Indian women. Fertil Steril 2009;91(2):432-439 DOI: 10.1016/j. fertnstert.2007.11.093

25. Lindgren I, Bååth $M$, Uvebrant $K$, et al. Combined assessment of polymorphisms in the LHCGR and FSHR genes predict chance of pregnancy after in vitro fertilization. Hum Reprod 2016;31(3):672-683. DOI: 10.1093/humrep/dev342

26. Perez Mayorga M, Gromoll J, Behre HM, et al. Ovarian response to follicle-stimulating hormone (FSH) stimulation depends on the FSH receptor genotype. J Clin Endocrinol Metab 2000;85(9):3365-3369. DOI: 10.1210/jcem.85.9.6789

27. Simoni M, Nieschlag E, Gromoll J. Isoforms and single nucleotide polymorphisms of the FSH receptor gene: implications for human reproduction. Hum Reprod Update 2002;8(5):413-421. DOI: 10.1093/humupd/8.5.413

28. Huang X, Li L, Hong L, et al. The Ser680Asn polymorphism in the follicle-stimulating hormone receptor gene is associated with the ovarian response in controlled ovarian hyperstimulation. Clin Endocrinol (Oxf) 2015;82(4):577-583. DOI: 10.1111/cen.12573

29. Behre HM, Greb RR, Mempel A, et al. Significance of a common single nucleotide polymorphism in exon 10 of the follicle-stimulating hormone $(\mathrm{FSH})$ receptor gene for the ovarian response to $\mathrm{FSH}$ : a pharmacogenetic approach to controlled ovarian hyperstimulation. Pharmacogenet Genomics 2005;15(7):451-456. DOI: 10.1097/01. fpc.0000167330.92786.5e 
30. Laven JS, Mulders AG, SuryandariDA, etal. Follicle-stimulating hormone receptor polymorphisms in women with normogonadotropic an ovulatory infertility. Fertil Steril 2003;80(4):986-992. DOI: 10.1016/s0015-0282(03)01115-4

31. Yan Y, Gong Z, Zhang L, et al. Association of follicle-stimulating hormone receptor polymorphisms with ovarian response in Chinese women: a prospective clinical study. PLoS One 2013;8(10):e78138. DOI: 10.1371/journal.pone.0078138

32. Dolfin E, Guani B, Lussiana C, et al. FSH-receptor Ala307Thr polymorphism is associated to polycystic ovary syndrome and to a higher responsiveness to exogenous FSH in Italian women. J Assist Reprod Genet 2011;28(10):925-930. DOI: 10.1007/s10815-011-9619-4

33. Lensen SF, Wilkinson J, Leijdekkers JA, et al. Individualised gonadotropin dose selection using markers of ovarian reserve for women undergoing in vitro fertilisation plus intracytoplasmic sperm injection (IVF/ICSI). Cochrane Database Syst Rev 2018;2(2): CD012693. DOI: 10.1002/14651858.CD012693.pub2

34. Drakopoulos P, Santos-Ribeiro S, Bosch E, et al. The effect of dose adjustments in a subsequent cycle of women with suboptimal response following conventional ovarian stimulation. Front Endocrinol 2018;9:361. DOI: 10.3389/fendo.2018.00361

35. Conforti A, Esteves SC, Di Rella F, et al. The role of recombinant $\mathrm{LH}$ in women with hypo-response to controlled ovarian stimulation: a systematic review and meta-analysis. Reprod Biol Endocrinol 2019;17(1):18. DOI: 10.1186/s12958-019-0460-4

36. Alviggi C, Conforti A, Esteves SC, et al. Recombinant luteinizing hormone supplementation in assisted reproductive technology: a systematic review. Fertil Steril 2018;109(4):644-664. DOI: 10.1016/j. fertnstert.2018.01.003

37. Lin $\mathrm{MH}, \mathrm{Wu} \mathrm{FS}$, Lee RK, et al. Dual trigger with combination of gonadotropin-releasing hormone agonist and human chorionic gonadotropin significantly improves the live-birth rate for normal responders in GnRH-antagonist cycles. Fertil Steril 2013;100(5):1296-1302. DOI: 10.1016/j.fertnstert.2013.07.1976

38. Haas J, Bassil R, Samara N, et al. GnRH agonist and hCG (dual trigger) versus $\mathrm{hCG}$ trigger for final follicular maturation: a double-blinded, randomized controlled study. Hum Reprod 2020;35(7):1648-1654. DOI: 10.1093/humrep/deaa107

39. Chern CU, Li JY, Tsui KH, et al. Dual-trigger improves the outcomes of in vitro fertilization cycles in older patients with diminished ovarian reserve: a retrospective cohort study. PLoS One 2020;15(7):e0235707. DOI: $10.1371 /$ journal.pone.0235707
40. Lin $\mathrm{MH}, \mathrm{Wu} F \mathrm{~S}, \mathrm{Hwu} \mathrm{YM}$, et al. Dual trigger with gonadotropin releasing hormone agonist and human chorionic gonadotropin significantly improves live birth rate for women with diminished ovarian reserve. Reprod Biol Endocrinol 2019;17(1):7. DOI: 10.1186/s12958-018-0451-x

41. Eftekhar M, Naghshineh E, Neghab N, et al. A comparison of dual triggering (by administration of GnRH agonist plus HCG) versus HCG alone in poor ovarian responders in ART outcomes. Middle East Fertil Soc J 2018;23(4):350-353.41. DOI: 10.1016/j. mefs.2018.04.011

42. Khalife D, Awwad J, Ghunaim S, et al. Dual triggering of final oocyte maturation in poor ovarian responders: a prospective randomized controlled trial. Fertil Steril 2019;112(3):e28-e29. DOI: 10.1016/j. fertnstert.2019.07.207

43. Farquhar C, Rombauts L, Kremer JA, et al. Oral contraceptive pill, progestogen or oestrogen pretreatment for ovarian stimulation protocols for women undergoing assisted reproductive techniques. Cochrane Database Syst Rev 2017;5(5):D006109. DOI: 10.1002/14651858.CD006109.pub3

44. Cozzolino M, Cecchino GN, Troiano G, et al. Growth hormone cotreatment for poor responders undergoing in vitro fertilization cycles: a systematic review and meta-analysis. Fertil Steril 2020;114(1):97-109. DOI: 10.1016/j.fertnstert.2020.03.007

45. Nagels HE, Rishworth JR, Siristatidis CS, et al. Androgens (dehydroepiandrosterone or testosterone) for women undergoing assisted reproduction. Cochrane Database Syst Rev 2015;26(11):CD009749. DOI: 10.1002/14651858.CD009749.pub2

46. Kamath MS, Mascarenhas M, Franik $S$, et al. Clinical adjuncts in in vitro fertilization: a growing list. Fertil Steril 2019;112(6):978-986 DOI: 10.1016/j.fertnstert.2019.09.019

47. Vaiarelli A, Cimadomo D, Ubaldi N, et al. What is new in the management of poor ovarian response in IVF?. Curr Opin Obstet Gynecol 2018;30(3):155-162. DOI: 10.1097/gco.0000000000000452

48. Vaiarelli A, Cimadomo D, Trabucco E, et al. Double stimulation in the same ovarian cycle (DuoStim) to maximize the number of oocytes retrieved from poor prognosis patients: a multicenter experience and SWOT analysis. Front Endocrinol 2018;9:317. DOI: 10.3389/fendo.2018.00317

49. Alsbjerg B, Haahr T, Elbaek HO, et al. Dual stimulation using corifollitropin alfa in 54 Bologna criteria poor ovarian responders-a case series. Reprod Biomed Online 2019;38(5):677-682. DOI: 10.1016/j. rbmo.2019.01.007 